\title{
AN OVERVIEW OF AMI FROM A USER CENTERED DESIGN PERSPECTIVE
}

\author{
Irene Mavrommati, John Darzentas \\ University of the Aegean, \\ Products and Systems Design Engineering department, Syros, Greece
}

\begin{abstract}
In this paper we will attempt to give an overview of issues related to $\mathrm{HCI}$ in AmI environments. We will address generic issues stemming from the nature of AmI research which relate to HCI practice, as well as specific HCI issues and shifts in current practice and paradigms due to new elements that are introduced by the very nature of living and interacting within AmI environments. This paper aims to raise awareness in the community of AmI researchers by attempting to provide a comprehensive overview of issues that relate to the current AmI HCI research.
\end{abstract}

\section{INTRODUCTION: THE GLOBAL FOCUS OF AMI RESEARCH}

Weiser (29) marked the vision of the future information society, by introducing the term Ubiquitous Computing (Ubicomp) and stating that in the third wave of computing each person will be "continually interacting with hundreds of nearby wirelessly connected computers" (Weiser, (30)). In the USA, IBM is using the term "pervasive computing" while Philips in Europe chose the term "Ambient Intelligence" (AmI) to describe the new paradigm for home computing and entertainment (Aarts and Marzano, (1)).

The European Union has been promoting the AmI vision within its research and development agenda by funding basic research initiatives and supporting policy creation for the forthcoming information society. Some examples are the Disappearing Computer initiative (IST/FET DC, 2001-2004) (DC (4)), the Situated and Autonomic Communications Initiative (SAC), the commissioning of reports aiming at policy-creation, such as Safeguards in the World of Ambient Intelligence (SWAMI) and Digital Territories (DT), in an attempt to pave the way to the Ambient Intelligence society. The European vision of "ambient intelligence" compared to Weiser's ubiquitous computing vision, is more towards "human centred computing"; Europe sees AmI as an integration of ubiquitous computing and communications with user centered design. While in the US and Europe the research focuses on miniaturisation and distribution of small networked computing units (i.e. the smart dust approach),
Japanese researchers are more interested in the ultimate user terminal, that is the one device that can do everything (SWAMI (23)).

\section{AMI VISION AND THE END USERS}

The highlights of the Ami vision is that computers will be everywhere (in objects of various sizes, from keys to cars, to buildings). These computers will be invisibly integrated into everyday life and will be supporting people in their activities, as diverse as these activities may be. The main components of this vision are (23):

o Reliable robust hardware with long lasting power supplies and of different sizes (possibly self managing or energy harvesting).

o Wireless and wired communications between computers, with collaborating different networks;

o Intuitive interfaces easily used by everybody (multimodal interfaces, that include various sensors as well as biometrics)

o Embedded intelligence capable reasoning about people unobtrusively, so as to provide them with services when needed, assist in controlling interfaces, and in from the systems perspective manage communications and maintenance (i.e. self-repairing).

This vision implies less direct and less conscious user input than the current systems.

There are two approaches regarding the visibility of AMI systems to end users - the comparison is notable to the early dispute in computing (between the merits of command language versus the merits of the desktop metaphor)- but these can be seen not necessarily as opposite, but rather as complementary:

a) People should not care about what's going on inside computers. This is generally based on the assumption that the AmI systems will be robust enough and error free, and intelligent agents will be based on the appropriate data and make appropriate judgements to appropriate actions).

b) People should be given a degree of transparency into the workings of the system. Transparency could be varied, according to the user and the context of use. (Markopoulos et al, (16)). AmI systems are offered with a recombinant constructivist approach aimed at end users 
(Newman et al (28), Rodden and Benford, (21), Mavrommati et al, (18). This transparency and user enabling model is promoted with the claim that via it AmI can achieve emerging niche applications -as a result of people's inherent creativity- (18), building of trust and thus adopting AmI systems, assistance with system failures and servicing, safeguarding privacy, etc.

It is obvious from the above that the human factor is a crucial element in the construction of an ambient intelligence world, and needs to be taken into account early in the research and development process of AmI systems. The success of ambient intelligence will depend on how individuals perceive AmI environments, whether they trust it and how willing they are to adopt and use AmI (via appropriate user experiences and interfaces). In turn people's trust depends on how secure the AmI world can be made, and how privacy and individual rights can be protected, as well as to the degree of trust to appropriate intelligent decisions made at the background of their attention (23). Trust is a vital factor for people to live uninhibitedly in AmI environments.

AmI manifests itself via wireless networks and electromagnetic fields around artefacts; potentially this may imply increased radiation on individuals. With the exception of the problematic raised by a few design-noir artefacts like the 'Faraday' and 'Nipple' chairs and the 'electro-drought excluder' ('Placebo objects' and 'Herzian Tales'), that raise the issue of the human concern and feeling of insecurity that may result increased living in electromagnetic radiation environments (Dunne and Raby, (6)), it has to be noted that health and safety issues pertaining living within pervasive AmI environments are not addressed, or mentioned as an issue.

\section{HIGH LEVEL AMI ISSUES THAT IMPACT HCI PRACTICE}

In this section we try to identify the high level issues of AmI research, that affect in turn HCI research practice in AmI. The problems identified in current AmI research seem to be in a nutshell:

o Fragmented vision of AmI

o Insufficient existing HCI practices, theories and models.

o Difficulties in the Evaluation process

o Multidisciplinary nature of research. (Current focus is too technology pulled/lead)

o Privacy and Security issues are poorly considered.

These will be addressed in more detail in the following paragraphs.

\section{Fragmented AmI vision}

There seems to be a lack of a concrete vision documentation relating to ubiquitous computing. There are, it seems, research agendas rather than vision documents that also contain some visions of the future (23). Nevertheless the vision for Ubicomp seems fragmented and this has consequences on the the lack of vision related to User Centered Design for AmI environments (a field that is still in it its infancy).

\section{Insufficient existing HCI practices in AmI}

Research main focus is on technology development that enables AmI (i.e. sensors technology, miniaturisation, processes, networking, middleware, energy provision), and not so much in the HCI issues occurring from AmI. There are insufficient existing HCI practices, theories and models regarding to $\mathrm{HCI}$ for AMI while User Centered Design is at its very early stages in this field of research.

We will outline this in more detail in the following section.

\section{Difficulties in the evaluation process}

In order to develop useful and usable AmI technology and applications, it is important that we are able to learn from results of research evaluations, from the user perspective. Evaluation here has to strike a delicate balance between prediction of how novel technologies will serve a real human need, and observation of actual use. (Dix et al, (5)) The coevolution of human activities and novel technologies plays a very important role here (Kameas and Mavrommati, (12)).

The fact that "there is surprisingly little research from an end user perspective in the Ubicomp community" as reported in the acknowledged HCI reference book of Dix, Finlay, Abowd and Beale. (5). According to them what can be applicable in AmI evaluation are participatory methods, design prompts using rich Ubicomp objects and large scale observation of subjects living and using the AmI world.

There are inherent difficulties in conducting evaluation for AmI systems, that relate to the difficulty of having robust prototypes of the application for evaluation relatively early in the progress of research. 'A good portion of reported ubicomp applications work remains at the level of demonstrational prototypes, not designed to be robust' as Dix et al, (5) notes. It is therefore difficult in AmI prototypical systems to evaluate for longer term, or with a large number of users. Even more difficult though it is to allow within the research process the time and effort for potential system changes that could be suggested by the evaluation feedback. Although some efforts are made to the direction of evaluating AmI prototypical systems (for 
example in the course of several DC projects (see: DC, e-Gadgets), it is noted that more extended evaluation is in fact needed (16).

To achieve more in-depth and extended evaluation some research organizations are creating AmI environment test-beds in the form of 'Living Labs' to support their research. Living labs seem to be a necessity for AmI research in order to achieve any longer term use and evaluation; a few such efforts are recorded, like the example of the Philips HomeLab (de Ruyter, (22)), or the Intelligent Dormitory at the University of Essex (iDorm), (Holmes et al, (9)).

Current evaluation results published are not very useful; this seems to be because evaluations are conducted in order to asses the quality of a particular AmI application, but also because there seems to be no shared framework and terminology being used in the field. While a number of evaluation methods and design guidelines can be borrowed from traditional $\mathrm{HCI}$, there are considerable differences that suggest different evaluation methodologies and metrics (Scholtz and Consolvo, (25)).

Belloti et al, (2) presented a model for interaction (that is more appropriate for the design of AmI interaction as a replacement of the widely used in traditional HCI Norman's "7 stages of execution" model) (as it is explain further in this paper), with an emphasis on communication rather than cognition. This framework, although it focuses in interaction, has potential in informing the design of the evaluation of AmI systems, (along with the design of AmI systems, as it was initially intended).

A more targeted effort towards a framework for evaluating AmI applications is published by Intel Research: Scholtz and Consolvo (25), suggest specific metrics for the following evaluation areas for AmI applications: Attention, Adoption, Trust, Conceptual models, Interaction, Invisibility, and Impact.

Formative evaluations are very important early on AmI research (due to the augmented cost and complexity involved in the development effort for AmI systems). In some cases existing evaluation methods such as Wizard of Oz can be used (25), or the Cognitive Dimensions framework (Green and Petre,(8)) -as reported in (16). The attempted framework of Intel Research (25) seems to propose more appropriate metrics for the evaluation of AmI systems. Nevertheless usability experts need to design the AmI evaluations carefully, starting from the basics, so as to apply appropriate metrics and method according to the AmI application at hand, as the field is not well established yet.

\section{Multidisciplinary nature of research}

The nature of AmI research is multidisciplinary, as it is clear from its research objectives. Nevertheless multidisciplinary AmI research is very often not practiced enough, or not practiced successfully. AmI research is by its nature technology lead; the research focus is technology-pulled while Humanities and Design are loosely integrated, if at all, in AmI research. Therefore, AmI research is not always well informed (especially regarding input from Sociology and Ethnography and a bit less so, Design and HCI). Even when the AmI research projects are multidisciplinary, because of the difficulties of crossdisciplinary goals and aims, and the subsequent gap in communication (Mackay, (15)), input from the different disciplines involved is not always well integrated in the research results.

\section{Privacy and security issues poorly addressed}

Privacy and Security issues are poorly considered in AmI . AmI research needs to start considering these issues early in research process. The European aim is to design technologies for people, instead of making people adapt to technology. Nevertheless it is ambient intelligence technology itself that violates most of currently existing privacy protecting borders. (26). Increased connectivity between people and spaces blurs the physical borders of operability (i.e. walls, doors). People tend to forget about the system being always 'on' and them potentially always being watched, especially because the intuitive expectation "If I can not see you, then you can not see me". Apart from the need to re-establish appropriate borders for Digital Territories, it is important to stress that there can be no guarantee that nobody can manipulate AmI systems for their own benefit (as is the case with most networked computing systems) (5).

To quote the SWAMI report (23):

'While the world of ambient intelligence will undoubtedly bring many benefits, trust and security should be designed into this world rather than inserted as an afterthought into an already constructed world of smart spaces. However, this goal is not possible to achieve in reality, at least not completely, in part because there are already islands of ambient intelligence and, in any event, the notion of "absolute security" is not feasible, as has been pointed by many experts. ....Thus, building trust and security into networks inevitably involves an effort of trying to create trustworthy systems from untrustworthy components. The success of this brave new world will depend on its acceptability by citizens and by taking steps to minimize their concerns with regard to how it might lead to further encroachments upon their privacy, safety and security.'

Ambient Intelligence, according to SWAMI report should be seen as an emerging property requiring a proper balance of a complex diversity of interests and values such as: protection of identity, of individual sphere, and against discrimination; as well as to be given the right of access to information and free expression. Trust is another parameter that needs to 
be taken into account ${ }^{1}$. Moreover the social, economic, legal and technological dimensions have to be taken into account, as well as the different AmI perspectives and definitions. Last but not least, technological innovations need to be addressed, the consequence of which are difficult to predict (i.e. the invisibility of networked "intelligent" devices, invisibility of system interacting actions, etc).

Palen and Dourish (20) attempted to propose a conceptual framework that would allow for more specific and detailed statements about privacy. In their view privacy management is a dynamic response to circumstance, rather than a static enforcement of rules. Privacy is seen as a balancing act, a resolution of tensions between people, their internal conflicting requirements, and the environment.

The Ambient Agoras DC project and Langheinrich,. (13) have developed a few 'Privacy Design Guidelines' that are useful to take into account when designing for Ambient Intelligent Environments. SWAMI report takes those guidelines into account, and concludes, with the following guidelines that can be generally applied in AmI research:

- Privacy considerations should be designed into the system from the start

- Privacy enhancing technologies should be easy to use and understand

- Individuals should be able to specify their privacy preferences

- Personal data should not be collected unnecessarily.

\section{SOME SPECIFIC HUMAN COMPUTER INTERACTION ISSUES FOR AMI}

\section{A few common assumptions}

Ami vision tents to present people as passive consumers accepting happily an increasing dependability on AmI systems. AmI sees people increasingly and comfortably relying on AmI for a number of activities (for reminders, surveillance, health monitoring, entertainment, home automation etc). Yet, it remains unclear how AmI systems are maintained. This vision does not seem realistic because AmI systems can not be absolutely problem free.

\footnotetext{
${ }^{1}$ We note here that we do not explicitly mention trust in this section, as we consider it as a more holistic aspect of In Scholtz and Consolvo's evaluation framework for Intel, Trust is considered an axis of their proposed evaluation framework, with it's metrics being Privacy and Awareness. (for more info see (Scholtz and Consolvo, 2004)).
}

SWAMI report mentions that it it is assumed that AmI technology is accepted by everybody and is available to everybody, and it is never considered, described or investigated what happens to people who are either not willing or not able to use Ami technology (23).

It is not clear whether new technologies will provide a means to escape gracefully from being always connected. (23), (16).

It is also questionable how people will accept living and evolving within AmI technological environments and how this 'nurture' will impact human nature (e.g., why train one's memory if AmI gives reminders?). (23). Social issues relate to how people's individual as well as social behavior will evolve by living in AmI environments, and thus developing certain expectations, assertions, and habits. (Kameas and Mavrommati, 12).

Some of the known HCI issues (5), that cannot easily be addressed within an AmI environment are:

- Visibility of action (feedback not visible)

- Reversibility of actions

- Syntactic correctness of actions

- Replacement at complex command languages with actions from manipulating directly the objects

Intelligence can help in the latter two of these actions; still there remain problems with its application in AmI, that will be described later.

\section{Intelligence as applied in AmI may cause HCI problems}

In the past, interaction design has focused on the user, who was explicitly telling the computer what to do. This is more implicit in the context aware computing, that is often inherent as an interaction method within AmI environments. Automatically sensing content is by its nature imperfect. It is therefore very important that actions resulting from so called 'intelligent' predictions are made with caution, based on the following two principles of applying intelligence appropriately (5):

0 be right as often as possible and useful assuming that the predictions are correct

o do not cause inordinate problems, in the events resulting of wrong predictions.

In AmI, action and language paradigm need to be combined. In the Action paradigm the use of agents is common. Agent based interfaces, can offer rules for certain kinds of actions. Agents can check for certain sequences of actions and suggest possible repetitions or next actions (5).

Nevertheless intelligence in an AmI environment should be applied carefully so that it does not change the AmI environment beyond the expectations of users (16): 
o Automation or adaptation actions should be visible and predictable, or at least justifiable.

o Intelligence should be applied only to simplify complex tasks. .

\section{A shift in the nature of interaction: to implicit interaction}

In AmI there is more than one user for an application. There are several organizational concerns exposed: of single users, but who may also operate in larger groups ${ }^{2}$. The input in AmI is distributed. Interaction within AmI can be conscious or not: the user may not always be aware that their action is in fact an interaction within the AmI environment.

In the Model-World metaphor for example (Hutchins et al, (10), the interface is itself 'a world where the user can act, and which changes the state in response to user actions. Appropriate use of the model world metaphor can create the sensation with the user of acting upon the objects of the task domain themselves. This aspect is called direct engagement'. (5). In AmI the direct engagement is not with the model world metaphor, but with the world itself. In the real word the input is physical, yet in the AmI world physical action gets converted to digital information too, and has consequences as an action of direct manipulation. Here the physical world becomes the interface of the AmI system, but is more than interface, as it cannot be separated (mentally or physically) from the physical environment.

As (5) puts it: In Ubicomp the user does not know where the computer is. It is the computing infrastructure that permeates over the physical environment, so that people do not notice it any longer. It is Utility that leads to invisibility (ubiquity), and hence the term Disappearing Computer used by the so called European initiative. The potential is what can be done with a number of objects in any place the user may be (be it the home, the car, or even one's own body). The user may be unaware where the interaction is taking place (i.e. via gestures, sensors, movement detectors, secret cameras). The implicit nature of interaction implied by sensing and physical action creates a human computer relationship so seamless that there may be no conscious interaction at all.

Thus, we are witnessing a radical shift in HCI: the basic models of interaction that have proved universal across technologies are questionable for AmI (5), (2), (25). Below we highlight the main changes from traditional HCI, as described in (5):

0 The focus becomes that of activities, rather than tasks.

o An emphasis is given to the design of continuously available interaction

\footnotetext{
${ }^{2} \mathrm{CSCW}$ research input can be valuable in understanding operation in groups.
}

o There are no starting or ending points for interaction in AmI environments

o Interruptions and Multiple actions can be in operations, that are loosely connected (no longer in terms of achieving certain goals).

o Multiple perspectives are on operation, that imply the reuse of information, for different functions (i.e. associative models of information are needed).

Systemic thinking is needed in order to approach Ami Environments. Theories such as Situated Action theory (Suchman, (27)), and Distributed Cognition theory (Lave, (14)) are being explored by the AmI community as being more appropriate in order to understand the interactions that take place in AmI environments. The above theoretical standpoint is that people do not act on the world, but act with the world. Thinking, according to Distributed Cognition theory, is not just within the head, but in the external relationships with things in the world and with other people. People are in constant dialog with the physical environment; they use this information stored in artefacts and their physical location to trigger and guide their actions (5). In this context incidental interaction that happens within an AmI environment has an importance that becomes considerable for the system design.

\section{The good old model's farewell}

As mentioned before, increased connectivity between people and spaces blurs the physical borders of observability, while the sheer quantity of information transfer makes this an impossible mental load to handle. Ubiquitous networks will need to track and collect significant data from users' activities. A pervasive network of interconnected devices and communications will mean that the sheer quantity of information in circulation will increase greatly, (26) and beyond the human perceptive capability (due to information overload).

AmI system design has to face grave challenges regarding the system's usage, as to how can users control the AmI system (i.e. with appropriate means, or aided by intelligent agent interfaces), how can they predict what the complex networked artefacts will do, and how the whole AmI system in turn will function appropriately and unobtrusively, providing for qualitative experience and safeguarding its user's privacy (i.e. avoiding stress and confusion in order to achieve actions, avoiding errors, and facilitating recovering from them).

A good case for study is Norman's (19) model of interaction that is widely used in HCI: the so called execution-evaluation cycle. The execution-evaluation cycle splits the interaction into a sequence of subactions, each of them being a result of a specific user intention. Initially the user is forming the goal, and then forming a sequence of intentions, followed by specific actions, the user proceeds in executing these 
actions, and perceiving the state of the system after these actions are performed (change state, communicated to the user via appropriate feedback). The user then interprets the new state of the system and finally evaluates the outcome comparing it to his/her initial goal (to what extent the goal is achieved). Belloti (2) attempts to re-think Norman's '7 stage' interaction model, focusing more in interactions that are are more appropriate for AmI environments (assuming the interactions are not GUI based). Belloti suggests the following five interaction challenges for AmI researchers, and exposes a subsequent number of design challenges, that designers should address:

1. Address - how to direct communication to a system)

o Disambiguate signal to noise

o Disambiguate intended target system

o How not to address the system

2. Attention - establishing that the system is attending

o Embody appropriate feedback, so that users are aware of system's attention

o Direct feedback to user's attention

3. Action - defining what can be done with the system

o Identify and select a possible interaction object

o Identify and select one action and bind it to the object(s) and avoid unwanted selection

o Handle complex operations (i.e. multiple objects and actions, more abstract functions)

4. Alignment - monitoring systems response.

o make the system state perceivable

o Direct timely and appropriate feedback

o Provide distinctive feedback on the response (results and state)

5. Accident - avoiding errors and misunderstandings, or, if they should happen, recovering from them.

o Control or cancel system action that is in progress

o The system intervenes when user makes obvious error

o Disambiguate what to undo, and do so in time.

Some possible pitfalls for AmI, that are identified in the Belloti model as possible problems, are: unintended actions, leading to undesirable results, failure to execute an action, limited operations available, wasted input effort in a non-attending system, inability to detect mistakes, difficulty in evaluating new state, inability to detect mistakes, and to recover the previous state.

\section{Interaction channels, input and output}

The input has changed. Forms adopt a variety of mobile devices up to even static surroundings. It has embraced artifacts not traditionally perceived as computers (i.e. the sensing wall, the floor), that may by themselves not have actuating capabilities (i.e. in the form of a screen).

The output may be ubiquitous and therefore may be not noticeable by end users (there may be no display, no sonic or visual or mechanical feedback present or may be away from the users focus of attention).

Unlike some parts of Weiser's vision (30), it may not be appropriate to the nature of many artefacts to have screens added to them, Such an interface approach applies to the specific category of information appliances, and although it is convenient for interaction, it does not always fit in the nature of objects and environments of the disappearing computer. The design of the object's form and physical properties will also affect the interaction. In fact the design of objects - which constitutes their interface - may have to be reconsidered so that their new capabilities can be promoted to the user (indicated by appropriate elements for the nature of each object) (17). In this broad picture, information appliances (Sharpe, (24)) as we know them are only a subset of these objects. Current information appliances are often screen-dominated. The AMI research agenda seems to impose screens everywhere (in very varied sizes, but in great abundance) into people's everyday environment. Yet, there is a subset of AmI objects that is beyond the point of on-screenfeedback. There are many examples of artifacts that may have sensors but not actuators (i.e. a floor or a table with added sensors, an object with a mere added RFID tag, etc), and therefore the screen implying an interface that conveniently leads the interaction with many information appliances- is not present. This can be a challenge for designers; this involves a holistic approach, whereby the tangible interface of the object not only provides for an optimal user-experience, but is also assuming the role of the interface to a larger set of interconnected causes and effects.

Most objects in our everyday lives have been designed for specific tasks; but this specificity constrains the ways in which we might use them. In general, everyday objects can be used in different ways, providing that the limits of their physical properties are not violated (17).

As objects become AmI objects and spaces become Digital Territories, people have to learn any new ways in which the can be use and inhabit them (that may have to be indicated by appropriate new affordances), as well as the tasks these might participate in. People will be interacting at the same time with individual objects and spaces as well as with the AmI applications of the environment. (17).

AmI environments may use audio, visual, haptic, kinaesthetic, biometric input, and have audio, visual, haptic, electromagnetic, mechanic output. The more holistic the input and output gets, the more complex the interaction approach becomes. Nevertheless this gives ample space for experimentation. Experimental interactive art thrives with using these opportunities, 
while experimentation in industrial design has brought many interesting results -often concerned about the nature of displays being ubiquitous, non obtrusive and aesthetically rich (i.e. Jereminco's dangling string, or the Tilting picture frame of Gaver).

As the interaction channels becomes more and more haptic and kinaisthetic, the interaction within AmI becomes richer. According to the availability -or notof the input/sensing channels and output/feedback channels the interaction within AmI ranges from intended to not intended and from conscious to not conscious.

\section{CONCLUTIONS: A FEW GUIDELINES FOR HCI CONSIDERATIONS IN AMI}

The paper aims to raise the awareness for HCI in AmI. It is addressed to AmI researchers of all related fields; (AmI computer system engineers designers and analysts, AmI communication network and AmI sensor network engineers, as well as HCI experts and AmI experience designers), attempting to give a comprehensive summative overview of issues that relate to the current AmI HCI research.

A selected few guidelines (based on the research issues outlined in more detail above), which can be seen as generic issues that need to be addressed in AmI research are:

\section{Regarding the process:}

1. Allow for consideration of HCI and privacy issues from the beginning of AmI research and in sufficient depth.

2. Plan adequately for AmI evaluations, with a stress on formative evaluations.

3. Address HCI issues inviting multidisciplinary interventions and perspectives.

\section{Regarding the System design and Interaction:}

1. Aim to create trustworthy systems from untrustworthy components

2. Allow for flexibility, levels of transparency for end users into the Ambient environment

3. Have appropriate and robust intelligence in place, or alternatively allow for the possibility of some user control, in the case she wants to have any. (in case things break down, etc)

4. Provide the means to escape gracefully (i.e. the 'off' switch).

5. Try to clarify interaction issues in order to address them later in detail, in the course of the design of the AmI system. A good starting point is the five interaction issues and subsequent exposed challenges as described in (2): Addressing the system, Attention, Action, Alignment, Accident.
Regarding the Vision:

1. A clear vision and HCI guidelines (in the form of a few basic principles for AmI interaction) is still needed to be established in the field. To establish it, it would take considerable targeted effort coupled with newly gained experience from working with AmI systems.

2. Stepping away from existing HCI models, or at least viewing them critically, is essential. The adoption of appropriate theories for AmI (such as Situated Action and Distributed Cognition theory) seems to be crucial to gaining a better understanding of AmI interactions on which to base research (5).

\section{REFERENCES}

1. Aarts E.,Marzano S. (2003). The new everyday: Views on Ambient Intelligence. Publisher: Uitgeverij 010, Rotterdam, the Netherlands.

2. Bellotti, V., Back, M., Edwards, W.K., Grinter, R.E., Henderson, A., Lopes, C., (2002). Making Sense of Sensing Systems: Five Questions for Designers and Researchers. In: Proceedings of the Conference on Human Factors and Computing Systems (2002) pp. 415-422

3. Digital Territories. Information at: http://cybersecurity.jrc.es/docs/DigitalTerritoryB ubbles.pdf

4. Disappearing Computer (DC) initiative. Information at: www.disappearing-computer.net

5. Dix A., Finlay J., Abowd G., Beale R. (2004) Human Computer Interaction, 3rd edition, Pearson / Prentice Hall publications.

6. Dunne T. and Raby F. (2001): the Placebo project (2000-2001) and Hertzian Tales (19961999) project, see http://www.dunneandraby.co.uk

7. Extrovert Gadgets (e-Gadgets) Projects, http://www.extrovertgadgets.net

8. Green TRG, Petre M (1996) Usability analysis of visual programming environments: a cognitive dimensions framework. J Vis Lang Comput 7:131-174

9. Holmes A, Duman H, Pounds-Cornish A (2002), The iDorm: Gateway to Heterogeneous Networking Environments, International ITEA Workshop on Virtual Home Environments, Paderborn, Germany.

10. Hutchins E., Hollan J., Norman, D. (1986) Direct Manipulation interfaces. In User Centered System Design, Norman D, Draper S. (ed). Lawrence Erlbaum Associates, Hilsdale, NJ pp. 87-124

11. i-Dorm: http://iieg.essex.ac.uk/idorm2/index.htm

12. Kameas A., Mavrommati I. (2003) Re-appearing interfaces of objects. Proceedings of the 10th International Conference on Human - Computer Interaction, (HCI International), Krete, Greece.

13. Langheinrich, M. (2001): Privacy by Design Principles of Privacy-Aware Ubiquitous Systems. In: Abowd, G. D.; Brumitt, B. et al. (Hrsg.): Proceedings of the Third International Conference on Ubiquitous Computing 
(UbiComp 2001). Berlin und Heidelberg,: Springer-Verlag (Lecture Notes in Computer Science, 2201), pp. 273-291.

14. Lave J. (1988) Cognition in practice: mind, mathematics, and culture in everyday life. Cambridge University press, Cambridge.

15. Mackay W. (2003). Educating Multidisciplinary Design Teams. In Tales of the Disappearing Computer, Hellenic Letters / CTI press publications, Patras, Greece.

16. Markopoulos P., Mavrommati I., Kameas A. (2004) End-User Configuration of Ambient Intelligence Environments: Feasibility from a User Perspective. EUSAI, European Symposium on Ambient Intelligence, Eindhoven, published in: Ambient Intelligence ISBN 3-540-23721-6 Springer Lecture Notes on Computer Science (LNCS3295), page 243-254

17. Mavrommati I., Kameas A. (2003). The evolution of objects into Hyper-objects: will it be mostly harmless? In Personal and Ubiquitous Computing. ACM, Volume 7, Numbers 3-4. Springer-Verlag London Ltd. pp: 176 - 181.

18. Mavrommati I., Kameas A. Markopoulos P. (2004). An editing tool that manages device associations in an in-home environment. In: Personal and Ubiquitous Computing. ACM, Springer-Verlag Volume 8, Numbers 3-4. pp: $255-263$.

19. Norman D. (1990). The design of everyday things, Doubleday, N.Y.

20. Palen L., Dourish P. (2003). Unpacking Privacy for a Networked World. In proceedings of CHI 2003, CHI Letters, Vol.no.5, Issue no. 1.

21. Rodden T, Benford S (2003) The evolution of buildings and implications for the design of ubiquitous domestic environments. In: Proceedings of the CHI 2003 conference on human factors in computing, Florida, USA, 5-10 April 2003
22. Ruyter, B. de (2005) Ambient Intelligence: visualising the future. In Advanced Visual Interfaces, (ACM).

23. Safeguards in a World of Ambient Intelligence (SWAMI), (2006). IST Deliverable D1: the brave new world of ambient intelligence: A state of the art review. Friedewald M. Vildjiounaite E., Wright D. (Ed). to be found in: http://swami.jrc.es

24. Sharpe B., (2001) Information Appliances: an introduction. Appliance Studio White Paper, at http://www.appliancestudio.com/publications/w hitepapers.htm

25. Scholtz J, Consolvo S. (2004). Towards a discipline for evaluating ubiquitous computing applications. INTEL white paper, IRS-TR-04004. To be found in: http://www.intelresearch.net/Publications/Seattle/022520041200 232.pdf

26. $\overline{\text { Situated and Autonomic Communications }}$ Initiative, http://www.cordis.lu/ist/fet/comms.htm

27. Suchman L (1987). Plans and situated actions: the problem of Human Machine Interaction. Cambridge University Press, Cambridge.

28. Newman W, Sedivy J, Neuwirth CM, Edwards $\mathrm{K}$, Hong JI, Izadi S, Marcelo K, Smith TF (2002) Designing for serendipity: supporting end-user configuration of ubiquitous computing environments. In: Proceedings of the conference on designing interactive systems (DIS 2002), London, UK, June 2002, ACM Press, pp 147156

29. Weiser, M., (1991) The Computer for the 21st Century,. Scientific American, 265, pp.94-104.

30. Weiser, M. (1993). Some Computer Science Issues in Ubiquitous Computing. CACM, 36(7), July $\quad 1993, \quad$ pp $\quad 75-84$ 
